\title{
A Robust Highly Aligned DNA Nanowire Array-Enabled Lithography for Graphene Nanoribbon Transistors
}

\author{
Seok Hee Kang, ${ }^{\dagger}$ Wan Sik Hwang, ${ }^{\S}$ Zhiqun Lin, ${ }^{*}, \|$ Se Hun Kwon, ${ }^{*}{ }^{\star}$ and Suck Won Hong ${ }^{*}, \dagger$
}

${ }^{\dagger}$ Department of Cogno-Mechatronics Engineering, Department of Optics and Mechatronics Engineering, and ${ }^{\ddagger}$ School of Materials Science and Engineering, Pusan National University, Busan 609-735, Republic of Korea

${ }^{\S}$ Department of Materials Engineering, Korea Aerospace University, Goyang 412-791, Republic of Korea

"School of Materials Science and Engineering, Georgia Institute of Technology, Atlanta, Georgia 30332, United States

Supporting Information
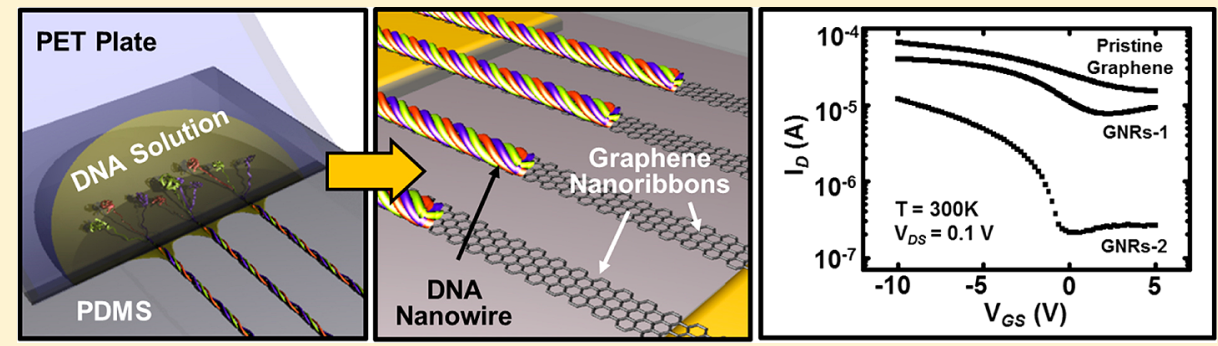

ABSTRACT: Because of its excellent charge carrier mobility at the Dirac point, graphene possesses exceptional properties for high-performance devices. Of particular interest is the potential use of graphene nanoribbons or graphene nanomesh for fieldeffect transistors. Herein, highly aligned DNA nanowire arrays were crafted by flow-assisted self-assembly of a drop of DNA aqueous solution on a flat polymer substrate. Subsequently, they were exploited as "ink" and transfer-printed on chemical vapor deposited (CVD)-grown graphene substrate. The oriented DNA nanowires served as the lithographic resist for selective removal of graphene, forming highly aligned graphene nanoribbons. Intriguingly, these graphene nanoribbons can be readily produced over a large area (i.e., millimeter scale) with a high degree of feature-size controllability and a low level of defects, rendering the fabrication of flexible two terminal devices and field-effect transistors.

KEYWORDS: DNA nanowire array-enabled lithography, self-assembly, graphene nanoribbon, transistor

$\mathrm{B}$ ecause of its exceptional electronic, thermal, optical, and mechanical properties, graphene, a single sheet of graphite, has received considerable attention since its discovery in 2004. ${ }^{1-4}$ Among various outstanding properties, graphene has been intensely explored to replace current Si-based materials for logic device applications as it possesses excellent charge carrier mobility at the Dirac point. However, the linear energymomentum dispersion behavior in graphene results in zero bandgap, which has an adverse effect and a poor $I_{\mathrm{ON}} / I_{\mathrm{OFF}}$ ratio. Notably, on the basis of theoretical calculation and experimental studies, the absence of such a bandgap in graphene can be overcome by a charge confinement effect in nanostructures. ${ }^{5-8}$ Recently, much effort has been concentrated on the fabrication of field-effect transistors employing graphene nanoribbons or graphene nanomesh and on the size confinement and the edge effect. ${ }^{9,10}$ When considering repeatability and controllability, electron-beam lithography is the most commonly used means of patterning graphene at tens-ofnanometer scale with smooth edge roughness. However, electron-beam exposure can lead to the irradiation damage to graphene as well as degrade its electronic transport property. ${ }^{11}$ Therefore, it is of key importance to identify alternative patterning methods to achieve nanoscale patterns on graphene without such an electron-beam irradiation effect. To date, several approaches have been invoked in the form of patterning technologies using nanoscale masks, such as block copolymers, nanowires, nanoimprinted polymer, and nanosphere lithography, followed by etching graphene sheets with oxygen plasma. ${ }^{10,12-18}$ Moreover, scalable graphene nanoribbons were produced by unzipping carbon nanotubes using various chemical synthesis approaches, ${ }^{19-21}$ direct growth from $\mathrm{SiC}^{22}$ and catalytic metal surface. ${ }^{23}$ Recently, a nanotemplate-based synthetic route to ultrathin graphitic ribbons directly on silicon wafers has also been reported. ${ }^{24}$

Self-assembly of nanoscale materials to form well-ordered structures promises a new opportunity to create useful engineered architectures and devices. It is worth noting that several simple and effective strategies based on evaporative selfassembly have emerged to produce complex yet ordered structures via irreversible solvent evaporation from a solution containing nonvolatile solutes, including polymers, nanoparticles, colloids, nanowires, biomaterials, and $\mathrm{DNA}^{25-33}$ for

Received: July 26, 2015

Revised: November 12, 2015

Published: November 16, 2015 
electronic, photonic, biological, and chemical-sensing applications. $^{34-38}$

Herein, we report on a robust strategy to create graphene nanoribbon transistors based on highly aligned DNA nanowire array-enabled lithography. First, an array of highly aligned DNA nanowires were crafted via flow-assisted self-assembly by subjecting the $\lambda$-DNA aqueous solution to evaporate in a restricted geometry consisting of a moving upper plate placed at the certain angle above the lower stationary plate made of flexible elastomer. Subsequently, the deposited DNA nanowires were transfer-printed onto a monolayer of graphene synthesized by thermal chemical vapor deposition (CVD), followed by oxygen plasma etching to remove graphene situated between adjacent $\lambda$-DNA nanowires (i.e., DNA nanowires served as shadow masks), thereby forming a large array of highly oriented graphene nanoribbons (GNRs) after the removal of DNA nanowires (i.e., a DNA nanowire array-enabled lithographic technique). Finally, a flexible two-channel device and bottomgated field-effect transistors were successfully fabricated by capitalizing on these parallel aligned arrays of GNRs formed by DNA nanowire array-enabled lithography.

DNA is a promising nanomaterial for constructing welldefined nanostructures for use in electronics, optoelectronics, sensors, etc. ${ }^{29}$ It possesses high aspect ratio with a diameter of 2 $\mathrm{nm}$ and a length of tens of micrometers. ${ }^{30}$ Figure 1 illustrates a
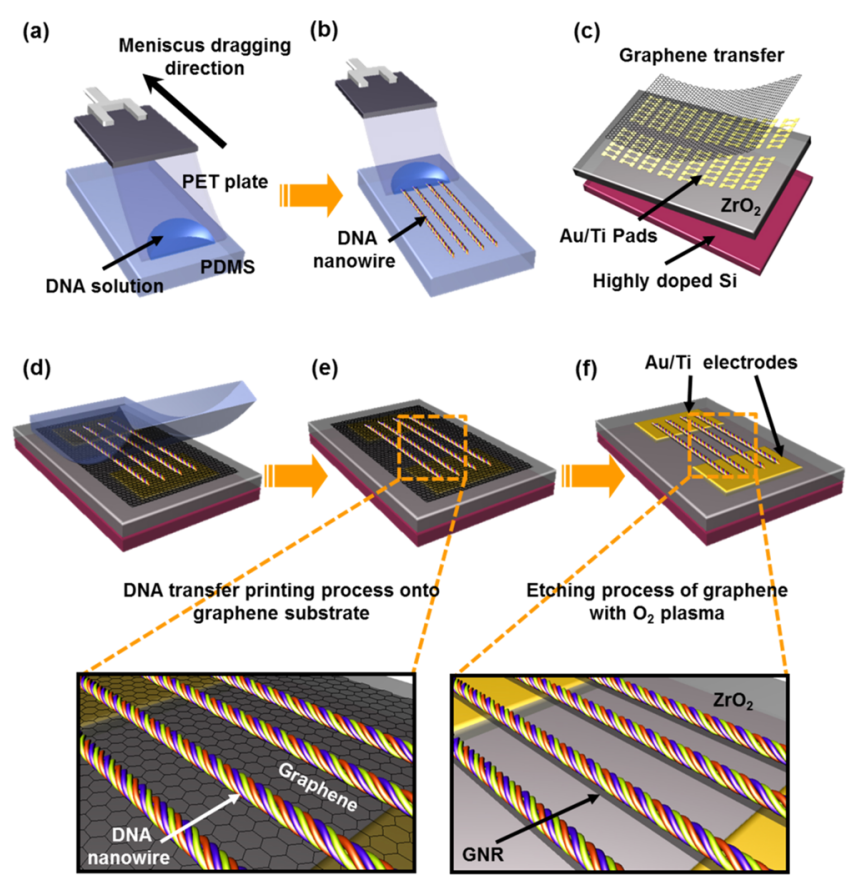

Figure 1. Stepwise representation of the formation of graphene nanoribbon-based field-effect transistors (GNR-FETs) based on highly aligned DNA nanowire array-enabled lithography. (a) Side view of a DNA aqueous solution trapped in a restricted geometry consisting of a motor-driven PET plate and a PDMS substrate. The PET plate was positioned above the flat PDMS substrate. (b) Formation of DNA nanowire array on the PDMS substrate by flow-assisted self-assembly. (c) Electric pads composed of $\mathrm{Au} / \mathrm{Ti}$ electrodes on the $\mathrm{ZrO}_{2}$ (gate dielectric)/heavily p-doped $\mathrm{Si}$ substrate. The CVD-grown graphene sheet was transferred onto the electric pads. (d) Transfer printing of inked DNA on PDMS stamp onto the graphene/electric pads substrate. (e) Gentle delamination of PDMS stamp allowing DNA inks to be deposited onto the graphene surface. (f) Oxygen plasma treatment for the removal of unprotected graphene region. schematic of a sequential process of flow-assisted self-assembly of bacteriophage $\lambda$-DNA (hereafter referred to as DNA), contact printing of the resulting DNA nanowire array, and formation of array of highly aligned GNRs by capitalizing on well-ordered DNA nanowires as mask. The first step involved the flow-assisted self-assembly of DNA molecules on a lower flat elastomeric PDMS stamp by dragging the DNA aqueous solution with a moving upper plastic plate made of poly(ethylene terephthalate) (PET) that was firmly attached to a motor-driven translation stage (Figure 1a,b). The upper PET plate was positioned at a certain angle above the lower flat PDMS substrate at a fixed gap of $\sim 10 \mu \mathrm{m}$ (Figure 1a), yielding a restricted geometry that constrained the drop of DNA solution (concentration $=50 \mu \mathrm{g} / \mathrm{mL}$ ). The PET plate was then moved to deposit and assemble DNA molecules into highly aligned nanowires (Figure $1 \mathrm{~b}) .{ }^{37}$ In the meantime, the electric pads were prepared by standard photolithography (AZ 5214) and lift-off process that defined $\mathrm{Au}(20 \mathrm{~nm}) / \mathrm{Ti}(5 \mathrm{~nm})$ patterned electrodes on the gate dielectric $\mathrm{ZrO}_{2}(50 \mathrm{~nm}) /$ heavily doped $\mathrm{Si}$ substrate for the bottom-gated transistor (Figure 1c). The CVD-grown graphene sheet was transferred to the electrode substrate noted above by poly(methyl methacrylate) PMMA-mediated transfer process (see Supporting Information) (Figure 1c). Subsequently, the aligned DNA nanowires (i.e., served as DNA "ink") on the PDMS substrate were transfer-printed onto the above-mentioned graphene surface by bringing this PDMS stamp in full contact with graphene for $2-3 \mathrm{~min}$ under a mild pressure $\left(\sim 0.2 \mathrm{~N} / \mathrm{m}^{2}\right)$ (Figure 1d). ${ }^{38}$ Pressing a soft elastomeric stamp against a graphene surface led to a conformal contact as a result of strong adhesion governed by van der Waals force and the molecular interaction. Then, a slow peeling-off of the flat PDMS stamp at sufficiently low velocity $(\sim 6 \mathrm{~mm} / \mathrm{min})$ successfully transferred the patterned DNA array onto the single layer of graphene substrate due to strong interaction between DNA and graphene (Figure 1e). Using a translation stage aligner, this transferprinting process can be effectively carried out and delivered DNA onto graphene that situated on the $\mathrm{Au} / \mathrm{Ti}$ electrodes with the DNA nanowires placed in the region between adjacent two terminal electrodes (Figure 1e and a close-up in the lower left panel). Finally, DNA nanowires were served as masks. The oxygen plasma treatment successfully removed graphene between DNA nanowires and exposed the gaps between adjacent $\mathrm{Au} / \mathrm{Ti}$ electrodes, thus providing an electrical isolation except GNRs that were formed directly under the DNA masks (Figure if and a close-up in the lower right panel).

Figure 2a describes the deposition process based on the flowassisted self-assembly of DNA molecules. Clearly, we developed a simple yet highly reproducible means of crafting a large-scale highly aligned fiber-like DNA array on the PMDS substrate without the necessity for preferential chemical modification of DNA (Figure 2b,c). As illustrated in Figure 2a, a drop of DNA solution $(15 \mu \mathrm{L}, 50 \mu \mathrm{g} / \mathrm{mL}$, diluted from the original solution in a Tris-EDTA buffer at $\mathrm{pH}=8.0$; New England Biolabs) was first loaded the restricted geometry as described above, forming a capillary-held solution at the zero moving speed of PET plate. Then, the DNA molecules jammed between hydrophobic PDMS substrate and flexible PET plate (PET thickness $=75$ $\mu \mathrm{m}$ ) and formed a three-phase contact line at the liquid-airsolid interface. Subsequently, the PET plate was moved slowly using a motor-driven translation stage at a speed of $6 \mathrm{~mm} / \mathrm{min}$; this led to the fingering instabilities at the drying front due to unfavorable interaction between DNA and PDMS (Figure 2a 
(a)

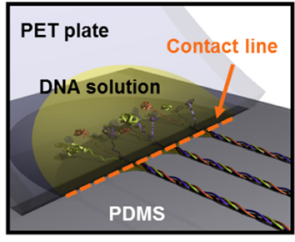

(b)

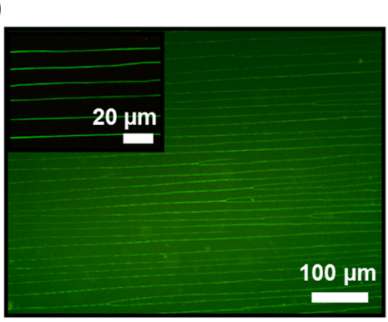

(d)

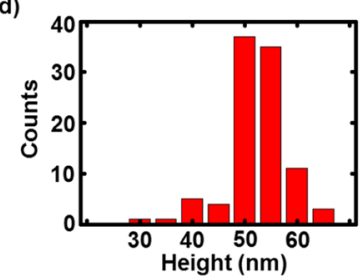

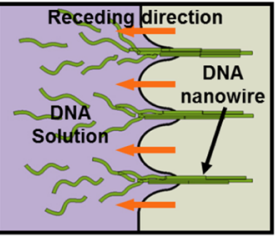

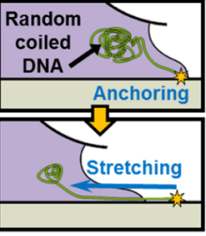

(c)

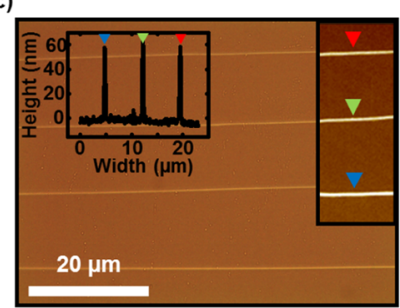

(e)

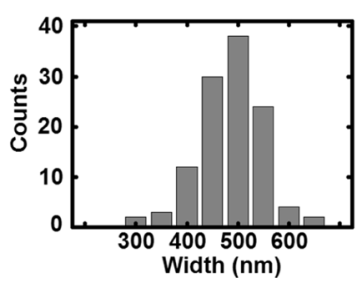

Figure 2. (a) Schematic illustration of the formation of highly aligned DNA nanowires on PDMS substrate by flow-assisted self-assembly (left panel); top view of the accumulation of DNA molecules at the receding meniscus during the moving of upper PET plate (middle panel); a side view of flow-assisted self-assembly where the anchoring and stretching of individual DNA molecules by the receding meniscus on the hydrophobic substrate (right panel). (b) A representative fluorescent micrograph of highly aligned DNA nanowires (greenemitting) on the PDMS substrate, obtained at a moving speed of PET plate of $6 \mathrm{~mm} / \mathrm{min}$. Scale bar $=10 \mu \mathrm{m}$ (inset $=100 \mu \mathrm{m})$. (c) AFM image of DNA array on the PDMS substrate (scale bar $=20 \mu \mathrm{m}$ ). The AFM height image is shown as an inset on the right. The average height of DNA nanowires is $60 \mathrm{~nm}$ (inset; left). (d) Histogram of the height of DNA nanowires on PDMS in a typical experimental condition $(\mathrm{pH}=8.0$, moving speed $=6 \mathrm{~mm} / \mathrm{min}$, angle between PET plate and PDMS substrate $=\sim 60^{\circ}$, and concentration $=\sim 50 \mu \mathrm{g} / \mathrm{mL}$ ). (e) Histogram of the width of DNA nanowires on the PDMS substrate under the same conditions.

and Figure S1). ${ }^{39}$ It has been reported that the extremities of randomly coiled DNA floating in solution anchored on the hydrophobic surface by exposing their hydrophobic core for adsorbing (i.e., a specific binding) over a certain range of $\mathrm{pH}$ values (right panel in Figure 2a). ${ }^{30,40}$ Such specific binding of DNA can resist the dragging force imposed by the motion of the PET plate. ${ }^{30}$ Thus, as the receding meniscus moved due to the motion of PET plate, the anchored DNA was stretched and acted as the nucleation site. More individual DNA molecules were then allowed to continuously transport and accumulate into aligned nanowires on the flat PDMS substrate (central panel in Figure 2a). ${ }^{41,42}$

Figure $2 \mathrm{~b}$ shows a representative fluorescence micrograph of an array of DNA nanowires formed by flow-assisted selfassembly at the moving speed of PET plate of $6 \mathrm{~mm} / \mathrm{min}$. Prior to the flow-assisted self-assembly process, DNA was stained with the fluorescent dye YOYO-1 (Molecular Probes, USA). Obviously, highly aligned DNA nanowires with remarkable homogeneity over a large area (i.e., large scale; up to several millimeters, depending on the size of PET plate used) were obtained (Figure $2 \mathrm{~b}$ ). It is noteworthy that this flow-assisted

self-assembly technique is fast, efficient, and highly reproducible for rapidly assembling DNA molecules along the moving direction of meniscus. ${ }^{43}$ In order to yield straight and parallel DNA nanowires which can serve as a functional resist for lithography, we optimized the temperature of PDMS substrate (i.e., $70{ }^{\circ} \mathrm{C}$ ) using a micro-hot plate during the receding of meniscus by moving the PET plate in order to accelerate the evaporation of water at the drying front of DNA solution (Figure S2). ${ }^{30,43}$ An optical micrograph in reflection mode of stretched DNA nanowires is shown in Figure 2c. The inset of Figure $2 \mathrm{c}$ shows the atomic force microscopy (AFM) height image and the corresponding cross-sectional height profile of uniformly deposited DNA nanowires, indicating that the average height and width of DNA nanowires are 50 and 450 $\mathrm{nm}$, respectively. Figures $2 \mathrm{~d}$,e depict the histograms of height and width of highly aligned DNA nanowire array, corresponding to the optical micrograph shown in Figure 2b, suggesting that DNA molecules were successfully immobilized and positioned on the PDMS substrate with a regular dimension.

To explore the formation of the array of DNA nanowires, we systematically investigated the correlation between the moving speed of PET plate and the density of stranded DNA nanowires on the substrate. Several different moving speeds of motordriven PET plate, ranging from 2 to $8 \mathrm{~mm} / \mathrm{min}$ at the fixed solution concentration $(c=50 \mu \mathrm{g} / \mathrm{mL})$, were applied. As shown in Figure $3 \mathrm{a}$, the density of DNA nanowires per specific
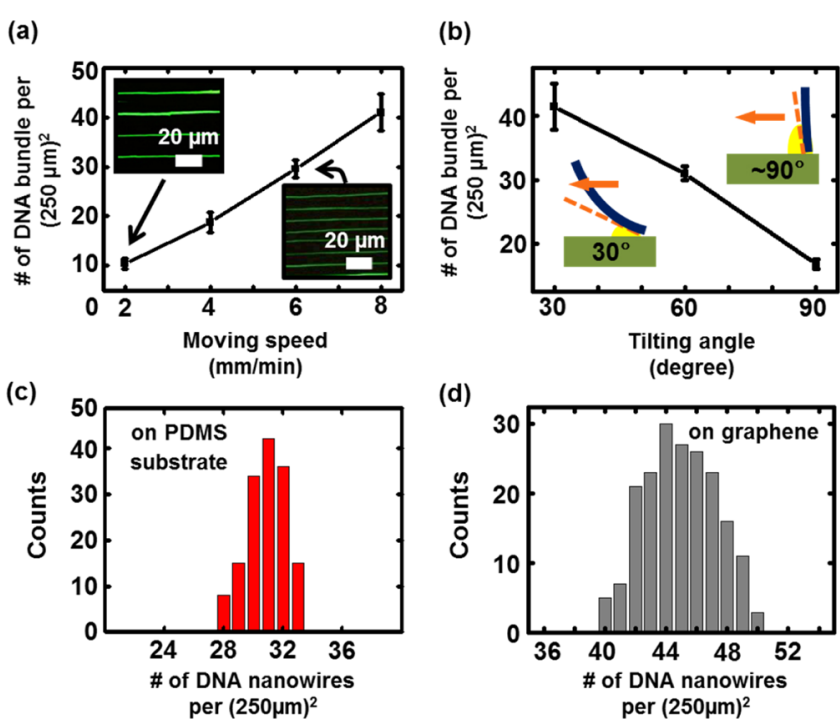

Figure 3. (a) Density of DNA nanowires as a function of moving speed of upper PET plate with an angle of $60^{\circ}$ between the tilted PET plate and the flat PDMS substrate. The representative fluorescence micrographs corresponding to 2 and $6 \mathrm{~mm} / \mathrm{min}$ of moving speed are shown as insets. (b) Density of DNA nanowires as a function of the tilting angle $\left(30^{\circ}, 60^{\circ}\right.$, and $\left.90^{\circ}\right)$ of PET plate with respect to PDMS substrate at the fixed moving speed of PET plate at $6 \mathrm{~mm} / \mathrm{min}$. Histograms of density of DNA nanowires (c) on PDMS substrate and (d) on CVD-grown graphene.

area (i.e., $250 \mu \mathrm{m} \times 250 \mu \mathrm{m}$ ) depends heavily on the moving speed. When the moving speed of PET plate increased, the anchored DNA molecules had less chance of converging with each other, and thus the possibility of accumulation of DNA molecules decreased, leading to the increased density of DNA nanowires as evidenced by the representative fluorescence micrographs in the insets of Figure 3a. The angle of PET plate 
(a)

(b)

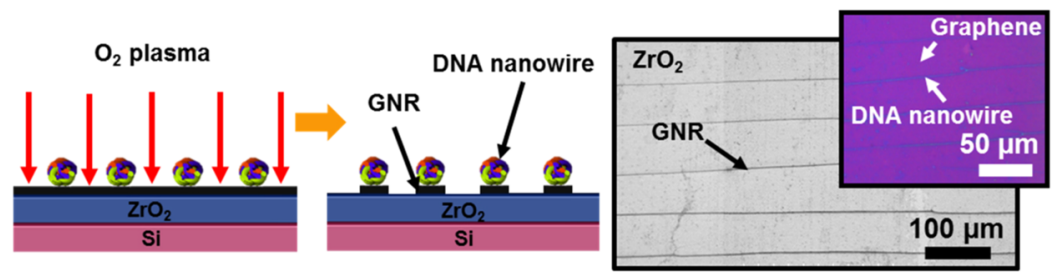

(c)

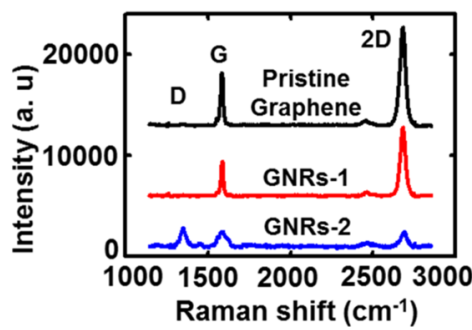

(d)

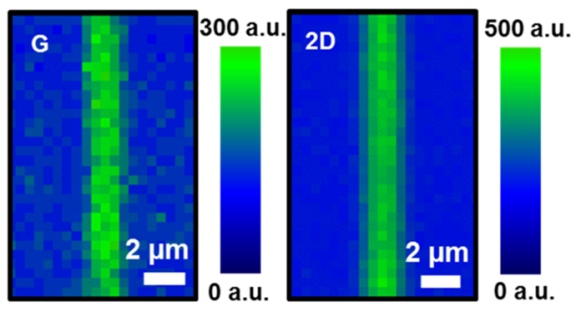

Figure 4. (a) Schematic illustration of highly aligned DNA nanowire array-enabled lithography for GNRs on $\mathrm{ZrO}_{2} / \mathrm{Si}$ substrate (cross-sectional view). (b) SEM image of highly aligned graphene ribbons obtained via etching using DNA nanowires as mask (scale bar $=100 \mu \mathrm{m}$ ). The optical micrograph of DNA nanowires transferred onto CVD-grown graphene is shown as the inset (scale bar $=50 \mu \mathrm{m}$ ). (c) Raman spectra for as-grown graphene sheet (black curve), GNRs-1 (red curve; width $=\sim 500 \mathrm{~nm}$ ), GNRs-2 (blue curve; width $=\sim 40 \mathrm{~nm}$ ) in the range of $1100-2900 \mathrm{~cm}^{-1}($ the excitation wavelength $=532 \mathrm{~nm}$ ). (d) Raman mapping of an GNRs-1.

with respect to the lower flat PDMS substrate were also adjusted (i.e., $30^{\circ}, 60^{\circ}$, and $90^{\circ}$ ) while moving the PET plate at a speed of $6 \mathrm{~mm} / \mathrm{min}$ to investigate the effect of the inclination of upper PET plate on the structure formation. As shown in Figure $3 \mathrm{~b}$, the observation revealed that the lower angle of moving PET plate led to an increase in the density of DNA nanowires. On the basis of optical microscopy measurements, when the angle between tilted PET plate and flat PDMS substrate was at $30^{\circ}$, the area of confined DNA aqueous solution was found to be approximately 3 times larger than that when they were nearly $90^{\circ}$ to each other. The larger interfaces of the confined DNA drop between PET plate and PDMS substrate resulted in a higher chance of DNA anchored near the three-phase contact line. Figures 3c,d summarize the histograms of density of aligned DNA nanowires on PDMS substrate and CVD-grown graphene. For the latter, DNA nanowires were directly formed on hydrophobic graphene substrate (i.e., not transfer-printing DNA formed on PDMS substrate onto CVDgrown graphene; Figure $\mathrm{S} 4 \mathrm{~b}$ ). It is interesting to note that other than PDMS, when polymers such as polystyrene (PS) and poly(methyl methacrylate) (PMMA) with a rms roughness of $0.5-1.4 \mathrm{~nm}$ were used as the flat substrate, the density and morphology of DNA nanowires on these substrates were similar to those on the PDMS substrate (i.e., perfectly aligned parallel to the direction of the moving plate as shown in Figure $2 \mathrm{~b})$. In contrast, in the case of CVD-grown graphene substrate, the intrinsic nanoscopic wrinkles and poor uniformity of synthesized graphene (rms roughness was 2.4-3.2 nm) originated from the roughened metal surface prohibited the uniform deposition of DNA molecules. As a result, DNA nanowires were not perfectly aligned with the emergence of branches and junctions (Figure S4b).

The resulting DNA nanowires on PDMS substrate were then exploited as the mask to yield GNR array by transfer-printing them onto CVD-grown graphene substrate, as illustrated in Figure 1d. Notably, unlike the use of a metallized $\mathrm{DNA}^{44}$ or an e-beam photoresist barrier ${ }^{45}$ as the etching mask, in this work the transfer-printed DNA nanowires were directly employed as the etching resist to create GNRs with high fidelity and regularity (see Supporting Information for optimized transferprinting). Such highly aligned DNA nanowire array-enabled lithography for GNRs dispenses with the need for the metallization step, thereby largely simplifying the fabrication process. Figure $4 \mathrm{a}$ illustrates a schematic of the oxygen plasma etching process in which the DNA nanowires array served as a nanostencil that defines the single-layer graphene sheet. After etching with mild oxygen plasma (100 W, $50 \mathrm{sccm}, 20 \mathrm{~s})$ followed by extensive washing to remove the DNA masks with a mixed solvent of DI water and isopropyl alcohol (IPA), a highly aligned GNR array was obtained, as clearly evidenced by scanning electron microscope (SEM) measurement, as shown in Figure $4 \mathrm{~b}$ with a length up to $1 \mathrm{~mm}$ in a high throughput manner which is advantageous for fabricating an active channel for devices. The optical micrograph in the inset of Figure $4 \mathrm{~b}$ shows DNA nanowires that lay on the top of single-layer graphene transferred onto a $\mathrm{ZrO}_{2} / \mathrm{Si}$ substrate synthesized by atomic layer deposition (ALD) (Figure 1e).

To examine the quality of GNRs, individual GNR was measured by Raman spectroscopy. Figure 4c displays the characteristic 2D $\left(\sim 2683 \mathrm{~cm}^{-1}\right), \mathrm{G}\left(\sim 1588 \mathrm{~cm}^{-1}\right)$, and D $\left(\sim 1352 \mathrm{~cm}^{-1}\right)$ peaks for pristine CVD-grown graphene and GNRs-1 $(w=\sim 500 \mathrm{~nm})$ and GNRs-2 $(w=\sim 20-60 \mathrm{~nm})$, respectively. ${ }^{46}$ For pristine graphene and GNRs-1, the $G$ and $2 \mathrm{D}$ bands had a reasonable intensity ratio $(<0.5)$, suggesting a mixture of mono- and bilayer graphene, regardless of mild oxygen plasma treatment. This signified the excellent shielding capability of DNA nanowires. In case of GNRs-2, however, Dband at $\sim 1345 \mathrm{~cm}^{-1}$ increased strongly during the oxygen plasma treatment, which may be due to the irregular edge disorders and oxidized dangling bonds of graphene. The position of the $2 \mathrm{D}$ peak in GNRs-2 shifted to $2705 \mathrm{~cm}^{-1}$ and the $G / 2 D$ ratio $(G / 2 D=\sim 1)$ increased as compared to that of untreated CVD-grown graphene $\left(\sim 2683 \mathrm{~cm}^{-1}, \mathrm{G} / 2 \mathrm{D}<0.5\right)$. These observations suggested some $\mathrm{p}$-doped graphene, which 
(a)

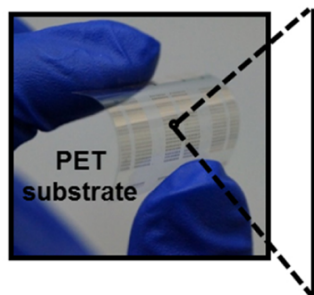

(b)

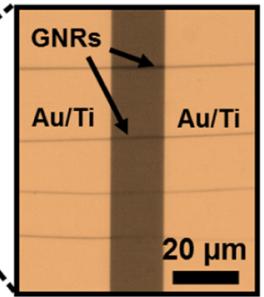

(d)

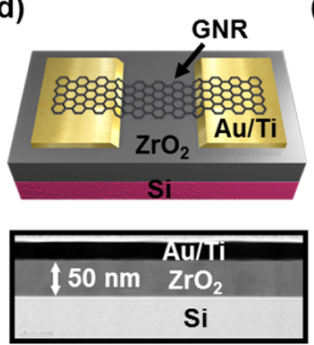

(e) (c)

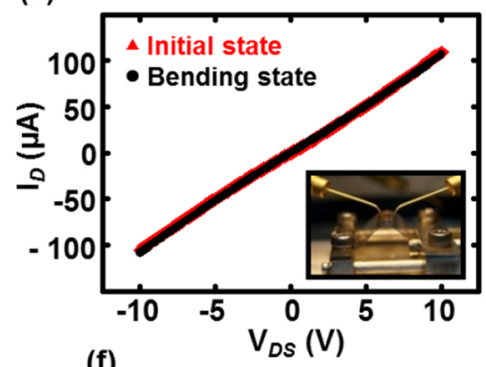

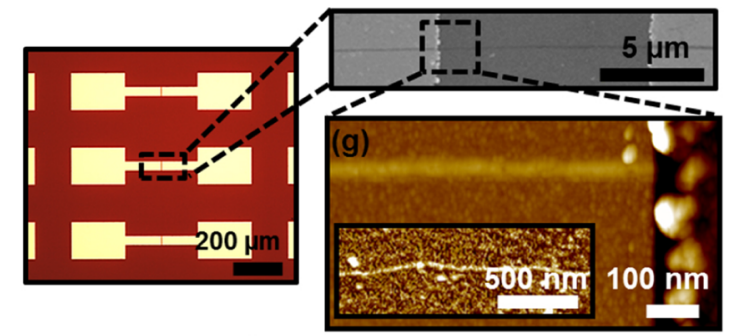

(h)

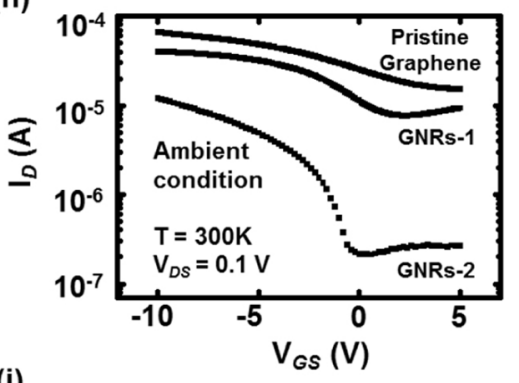

(i)

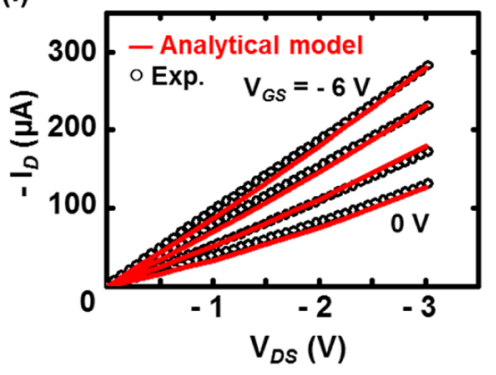

(1)
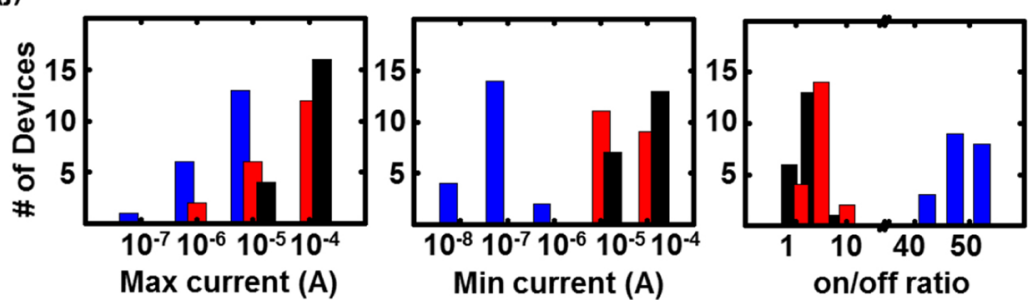

Figure 5. (a) Digital image of a flexible, two-channel electrode ( $\mathrm{Au} / \mathrm{Ti}$ ) array on a flexible PET substrate. (b) Optical micrograph of a close-up region of pairs of electrodes in (a); highly aligned DNA arrays were perfectly transferred onto the graphene/electrode/PET substrate. (c) $I-V$ characteristics of representative flexible two-channel device with graphene nanoribbons after oxygen plasma process before (triangles) and after (circles) the bending of PET (bending radius $\sim 1 \mathrm{~mm}$ ); the lines are the corresponding linear fits. (d) Schematic illustrating the cross section of a bottom-gated transistor, where GNR was allowed to be sit on a pair of electrodes (i.e., source and drain) with conformal bottom contacts with Au (upper) (upper panel); TEM image of the structure of a GNR-FET (lower panel). (e) Optical micrograph of GNR-FETs. (f) SEM image of a GNR between two electrodes (scale bar $=5 \mu \mathrm{m}$ ). (g) AFM image of a close-up marked in (f), showing an individual aligned GNR (the width, $W_{\mathrm{GNR}}=\sim 40$ $\mathrm{nm}$ ). In contrast, an AFM image of an individual DNA that was directly deposited on CVD-grown graphene, showing the possibility of fabricating a narrower GNR $\left(W_{\mathrm{GNR}}=\sim 20 \mathrm{~nm}\right.$, scale bar $=500 \mathrm{~nm}$ ) (inset). (h) Typical transfer characteristics of three different width range of GNRs $\left(L_{\mathrm{C}}=10\right.$ $\mu \mathrm{m})$ for a source/drain bias. Drain current $\left(I_{\mathrm{D}}\right)$ as a function of gate voltage $\left(V_{\mathrm{GS}}\right)$ for a source/drain bias $\left(V_{\mathrm{DS}}\right)$ of $0.1 \mathrm{~V}$ measured on a bottomgated transistor that incorporated the aligned array of GNRs. (i) Analytical model (red line) and measured drain current, $I_{\mathrm{D}}$ (open circles), at different $V_{\mathrm{GS}}\left(-6\right.$ to $0 \mathrm{~V}$ from the top with a $2 \mathrm{~V}$ interval) as a function of $V_{\mathrm{DS}}$ measured at room temperature in air. (j) Histograms of the maximum current, the minimum current, and the on/off ratio extracted from the transfer characteristics from 20 devices each for pristine graphene (black bars), GNRs-1 (red bars), and GNRs-2 (blue bars), respectively.

was due likely to the intrinsic large edge effect of narrow $\mathrm{GNR}^{16,47-49}$ Moreover, as compared to that of the original CVD-grown graphene film, there was a wide G-band in the GNRs-2 Raman spectrum; this also substantiated that narrow GNR was successfully fabricated from highly aligned DNA nanowire array-enabled lithography. Interestingly, the representative Raman mapping of $2 \mathrm{D}$ and $\mathrm{G}$ peaks (Figure $4 \mathrm{~d}$ ) showed the disappearance of unprotected graphene region, implying the effective removal of graphene exposed between transfer-printed DNA nanowires (Figure 4d).
We note that the transfer printing of highly aligned DNA nanowire array obtained from flow-assisted self-assembly is highly versatile. The aligned DNA nanowires can also be successfully printed on flexible PET substrate. The surface of flexible PET was predeposited with pairs of $\mathrm{Au}(20 \mathrm{~nm}) / \mathrm{Ti}$ (5 $\mathrm{nm}$ ) electrodes, followed by the CVD-grown graphene. As shown in Figures $5 \mathrm{a}$ and $5 \mathrm{~b}$, when using bottom contact $\mathrm{Au} / \mathrm{Ti}$, which is relatively thin $(25 \mathrm{~nm})$, the deposition (i.e., transfer) of graphene and the transfer printing of self-assembled DNA nanowires was successfully performed without the feature 
relaxation or a serious loss of resolution. ${ }^{50}$ The representative $I-V$ curves of two channel devices, before (red triangles) and after (black circles) bending of PET substrate, are shown in Figure $5 c$, where the solid lines are the linear fits. Moreover, an array of bottom-gated GNR-based field-effect transistors (GNR-FETs) was constructed. The top panel in Figure 5d illustrates the side view of GNR-FET; a corresponding transmission electron microscopy (TEM) image is shown in the lower panel. Figure 5e shows optical micrograph of electric pads of the bottom contact substrate with the GNRs aligned parallel to the channel length. In this work, a layer of $\mathrm{ZrO}_{2}$ (50 $\mathrm{nm})$ and $\mathrm{Au} / \mathrm{Ti}(25 \mathrm{~nm})$ was used as the gate dielectric and the source/drain electrodes, respectively (lower panel in Figure $5 \mathrm{~d})$. A SEM image showing the channel region of the device is shown in Figure 5f. Notably, we fabricated a multichannel GNR device with excellent alignment based on the process shown in Figure S5. A close examination by AFM measurement revealed that the average width of each GNR was approximately $\sim 40 \mathrm{~nm}$ obtained after extensive oxygen plasma treatment (Figure $5 \mathrm{~g}$ ). It is noteworthy that the inset in Figure 5g shows that instead of the transfer printing process of DNA, a DNA nanowire was directly deposited on CVD-grown graphene, yielding a much narrower GNR ( $\sim 20 \mathrm{~nm}$ as compared to $\sim 40 \mathrm{~nm}$ obtained from the transfer printing of DNA nanowire). However, irregular patterns were emerged due to the presence of intrinsic surface wrinkles of CVD-grown graphene (Figure S4) as noted above, which were often observed in previous studies. ${ }^{16,45-47}$

Figure $5 \mathrm{~h}$ presents the room temperature transfer characteristics of representative transistors for three different ranges of GNR widths at the same ribbon length. Characterization of the electrical responses, that is, the drain current $\left(I_{\mathrm{D}}\right)$ as a function of grate voltage $\left(V_{\mathrm{G}}\right)$ of GNR-FETs for a source/drain bias $\left(V_{\mathrm{DS}}\right)$ of $0.1 \mathrm{~V}$, revealed the key properties and provided some useful insight into graphene ribbons aspects of the width range. We observed that the gate modulation in the log scale is inversely proportional to the width of GNR. While the pristine graphene and GNRs-1 devices exhibited a weak gate modulation showing a high on-current, GNRs-2 delivered a less current in the on-state but showed a significantly improved $I_{\mathrm{ON}} / I_{\mathrm{OFF}}$ ratio. The $I_{\mathrm{ON}} / I_{\mathrm{OFF}}$ ratio of the GNRs- 2 with a width of $\sim 40 \mathrm{~nm}$ was approximately 56 at the identical gate stacks and $V_{\mathrm{BG}}$ window, suggesting the opening of a bandgap in GNRs-2 due to the charge carrier confinement effect in the transverse direction and the edge effect when the width of GNR is reduced. ${ }^{8}$ It is notable that these results are comparable to those recently reported in the literature, ${ }^{18,46}$ thus providing a critical evidence on the impact of biologically inspired DNA etch-mask technique on the nanoscale ribbons fabrication without any noticeable device degradation. The Dirac point of GNRs- 1 appeared at the positive region of $V_{\mathrm{GS}}$, whereas that of GNRs-2 appeared near $0 \mathrm{~V}$, which also indicated the presence of a bandgap in GNRs-2 whose edge is easily oxidized and influenced by surface adsorbates. This resulted in a p-doping effect for the GNRs-2 channel on the oxide. ${ }^{16,47-49}$ In this work, the extracted band gap of GNRs-2 is expected to be $\sim 20$ $\mathrm{meV}$ from the relationship between the width and energy bandgap of GNR, ${ }^{8}$ which was not sufficient to suppress thermal emission current at room temperature, and thus resulted in a high $I_{\mathrm{OFF}}$ current and low $I_{\mathrm{ON}} / I_{\mathrm{OFF}}$ ratio. Further scaling down of DNA nanowire can lead to GNRs with the narrower width and higher band gap. From the transfer curve, the linear-regime mobility, $\mu$ can be calculated using the peak transconductance at a source/drain bias $\left(V_{\mathrm{DS}}\right)$ of $0.1 \mathrm{~V}$. The mobility of multichannel GNRs-FET was estimated to be $\sim 450 \mathrm{~cm}^{2} /(\mathrm{V} \mathrm{s})$, based on $\mu=L_{\mathrm{c}} g_{\mathrm{m}} / W_{\mathrm{GNR}} C_{\mathrm{g}} V_{\mathrm{DS}}$, where $C_{\mathrm{g}}$ is the gate capacitance, $g_{\mathrm{m}}$ is the transconductance, $L_{\mathrm{c}}$ is the channel length, and $W_{\mathrm{GNR}}$ is the statistically averaged mean width of the GNRs. ${ }^{51}$ A family of $I_{\mathrm{D}}-V_{\mathrm{DS}}$ curves at various $V_{\mathrm{GS}}$ was shown and correlated with the analytical model of a traditional $\mathrm{Si}$ long channel (Figure 5i), according to the following equation: ${ }^{52}$

$$
\begin{aligned}
& I_{\mathrm{D}}= \\
& \frac{e \mu(W / L) \int_{I_{\mathrm{D}} R_{\mathrm{c}}}^{V_{\mathrm{D}}-I_{\mathrm{D}} R_{\mathrm{c}}} \sqrt{n_{\mathrm{O}}^{2}+\left[C_{\mathrm{OX}}\left(V_{\mathrm{GS}}-V_{\mathrm{DP}}-V(x)\right) / e\right]^{2}} \mathrm{~d} V(x)}{1+\mu\left(V_{\mathrm{DS}}-2 I_{\mathrm{D}} R_{\mathrm{c}}\right) / L v_{\mathrm{SAT}}}
\end{aligned}
$$

where the hole mobility $\mu_{\text {Hole }}=\sim 500 \mathrm{~cm}^{2} /(\mathrm{V} \mathrm{s})$, the contact resistance $R_{\mathrm{c}}=\sim 500 \Omega$, the gate capacitance $C_{\mathrm{OX}}=2.7 \times 10^{-7}$ $\mathrm{F} / \mathrm{cm}^{-2}$, the saturation velocity $\nu_{\mathrm{SAT}}=1 \times 10^{8} \mathrm{~cm} / \mathrm{s}$, and the residual charge density $n_{\mathrm{O}}=2 \times 10^{11} \mathrm{~cm}^{-2}$. Similar to other narrow band gap semiconductors, the parameters used in the model were comparable and fit well with the measured ones in the linear region, revealing that the fitting parameters in the model corresponded to the dominant physical mechanisms. The extracted residual charges in GNRs- 2 on oxides prepared using DNA nanowires as mask were comparable to the reported values of $(2-5) \times 10^{11} \mathrm{~cm}^{-2}$. 33,54 Clearly, the use of DNA nanowires as mask did not introduce additional impurities in GNR-FETs (Figure S5). The histograms in Figure $5 \mathbf{j}$ summarize the statistics on the device properties (20 devices each for pristine graphene (black bars), GNRs-1 (red bars), and GNRs-2 (blue bars)). The metrics for comparison include the maximum on-current, the minimum off-current, and the $I_{\mathrm{ON}} / I_{\mathrm{OFF}}$ ratio at the same grate voltage $\left(V_{\mathrm{G}}\right)$ range. One possible reason for the variations observed here may be associated with the spatial variations in the chirality of GNRs along with the edges. However, it is clear that our studies demonstrate that the on/off current ratio of GNRs can be significantly improved upon narrowing the width of GNRs to achieve a largest on/off current ratio at room temperature.

In summary, we developed a robust strategy based on highly aligned DNA nanowire array-enabled lithography to create graphene nanoribbon transistors. The array of highly oriented DNA nanowires was crafted by flow-assisted self-assembly of the DNA aqueous solution from a restricted geometry consisting of a moving PET plate and a stationary PDMS substrate. Subsequently, DNA nanowires were employed as "ink" and transfer-printed onto graphene sheet with high fidelity. The transferred DNA ink served as lithographical resist upon oxygen plasma exposure, thereby yielding highly aligned graphene nanoribbon array that was capitalized on to fabricate graphene nanoribbon transistors and flexible two terminal devices. On the basis of this work, it is clear that highly aligned DNA nanowires array-enabled lithographical technique offers new opportunities to realize the potential of DNA-based nanotechnology and nanodevices, which judiciously combine DNA as either scaffold or templates with a set of functional nanomaterials. Looking to the future, the combination of the DNA etch-masking approach with anisotropic etching technique may provide a viable solution to fabricating highly aligned GNRs with defined chiral edges. ${ }^{55}$ Moreover, we envision that follow-up studies on the cleanly etched graphene nanoribbons over a large area (i.e., millimeter scale) with a high degree of feature-size controllability and a low level of defects can be effectively fabricated to yield a wide variety of functional 
devices that are useful for exploring their physical properties and potential practical applications.

\section{ASSOCIATED CONTENT}

\section{S Supporting Information}

The Supporting Information is available free of charge on the ACS Publications website at DOI: 10.1021/acs.nanolett.5b02946.

Methods, Figures S1-S5 (PDF)

\section{AUTHOR INFORMATION}

\section{Corresponding Authors}

*E-mail swhong@pusan.ac.kr (S.W.H.).

*E-mail sehun@pusan.ac.kr (S.H.K.).

*E-mail zhiqun.lin@mse.gatech.edu (Z.L.).

\section{Notes}

The authors declare no competing financial interest.

\section{ACKNOWLEDGMENTS}

This work was supported by National Research Foundation of Korea Grant funded by the Korean Government (NRF2014R1A1A2058350) and National Science Foundation (NSF CBET-1332780).

\section{REFERENCES}

(1) Novoselov, K. S.; Geim, A. K.; Morozov, S. V.; Jiang, D.; Zhang, Y.; Dubonos, S. V.; Grigorieva, I. V.; Firsov, A. A. Science 2004, 306, 666-669.

(2) Han, T.-H.; Lee, Y.; Choi, M.-R.; Woo, S.-H.; Bae, S.-H.; Hong, B. H.; Ahn, J.-H.; Lee, T.-W. Nat. Photonics 2012, 6, 105-110.

(3) Bae, S.; Kim, H.; Lee, Y.; Xu, X.; Park, J.-S.; Zheng, Y.; Balakrishnan, J.; Lei, T.; Ri Kim, H.; Song, Y. I.; Kim, Y.-J.; Kim, K. S.; Ozyilmaz, B.; Ahn, J.-H.; Hong, B. H.; Iijima, S. Nat. Nanotechnol. 2010, 5, 574-578.

(4) Yan, Z.; Lin, J.; Peng, Z.; Sun, Z.; Zhu, Y.; Li, L.; Xiang, C.; Samuel, E. L.; Kittrell, C.; Tour, J. M. ACS Nano 2012, 6, 9110-9117.

(5) Li, X.; Wang, X.; Zhang, L.; Lee, S.; Dai, H. J. Science 2008, 319 (5867), 1229-1232.

(6) Son, Y. W.; Cohen, M. L.; Louie, S. G. Phys. Rev. Lett. 2006, 97, 216803.

(7) Chen, Z.; Lin, Y.-M.; Rooks, M. J.; Avouris, P. Phys. E 2007, 40 (2), 228-232.

(8) Han, M. Y.; Ozyilmaz, B.; Zhang, Y. B.; Kim, P. Phys. Rev. Lett. 2007, 98, 206805/1-206805/4.

(9) Kosynkin, D.; Higginbotham, A.; Sinitskii, A.; Lomeda, J.; Dimiev, A.; Price, K.; Tour, J. Nature 2009, 458, 872-876.

(10) Bai, J.; Zhong, X.; Jiang, S.; Huang, Y.; Duan, X. Nat. Nanotechnol. 2010, 5, 190-194.

(11) Childres, I.; Jauregui, L. A.; Foxe, M.; Tian, J.; Jalilian, R.; Jovanovic, I.; Chen, Y. P. Appl. Phys. Lett. 2010, 97, 173109.

(12) Kim, J. Y.; Kim, B. H.; Hwang, J. O.; Jeong, S.-J.; Shin, D. O.; Mun, J. H.; Choi, Y. J.; Jin, H. M.; Kim, S. O. Adv. Mater. 2013, 25, 1331-1335.

(13) Son, J. G.; Son, M.; Moon, K. J.; Lee, B. H.; Myoung, J. M.; Strano, M. S.; Ham, M. H.; Ross, C. A. Adv. Mater. 2013, 25, 47234728.

(14) Bai, J.; Duan, X.; Huang, Y. Nano Lett. 2009, 9, 2083-2087.

(15) Liao, L.; Bai, J.; Lin, Y. C.; Qu, Y.; Huang, Y.; Duan, X. Adv. Mater. 2010, 22, 1941-1945.

(16) Sinitskii, A.; Tour, J. M. Appl. Phys. Lett. 2012, 100, 103106.

(17) Liang, X.; Wi, S. ACS Nano 2012, 6, 9700-9710.

(18) Liu, L.; Zhang, Y. L.; Wang, W. L.; Gu, C. Z.; Bai, X. D. Adv. Mater. 2011, 23, 1246-1251.

(19) Kosynkin, D.; Higginbotham, A.; Sinitskii, A.; Lomeda, J.; Dimiev, A.; Price, K.; Tour, J. Nature 2009, 458, 872-876.
(20) Jiao, L. Y.; Zhang, L.; Wang, X. R.; Diankov, G.; Dai, H. J. Nature 2009, 458, 877-880.

(21) Jiao, L. Y.; Wang, X. R.; Diankov, G.; Wang, H. L.; Dai, H. J. Nat. Nanotechnol. 2010, 5, 321-325.

(22) Sprinkle, M.; Ruan, M.; Hu, Y.; Hankinson, J.; Rubio-Roy, M.; Zhang, B.; Wu, X.; Berger, C.; de Heer, W. A. Nat. Nanotechnol. 2010, 5, 727-731.

(23) Kato, T.; Hatakeyama, R. Nat. Nanotechnol. 2012, 7, 651-656.

(24) Sokolov, A. N.; Yap, F. L.; Liu, N.; Kim, K.; Ci, L.; Johnson, O. B.; Wang, H.; Vosgueritchian, M.; Koh, A. L.; Chen, J.; et al. Nat. Commun. 2013, 4, 2402.

(25) Hong, S. W.; Byun, M.; Lin, Z. Q. Angew. Chem., Int. Ed. 2009, $48,512-516$.

(26) Hong, S. W.; Wang, J.; Lin, Z. Q. Angew. Chem., Int. Ed. 2009, $48,8356-8360$.

(27) Hong, S. W.; Xu, J.; Xia, J.; Lin, Z. Q.; Qiu, F.; Yang, Y. L. Chem. Mater. 2005, 17, 6223-6226.

(28) Hong, S. W.; Jeong, W.; Ko, H.; Kessler, M. R.; Tsukruk, V. V.; Lin, Z. Q. Adv. Funct. Mater. 2008, 18, 2114-2122.

(29) Li, B.; Han, W.; Byun, M.; Zhu, L.; Zou, Q.; Lin, Z. ACS Nano 2013, 7 (5), 4326-4333.

(30) Li, B.; Zhang, C.; Jiang, B.; Han, W.; Lin, Z. Angew. Chem., Int. Ed. 2015, 54, 4250 .

(31) Lin, Y.; Balizan, E.; Lee, L. A.; Niu, Z.; Wang, Q. Angew. Chem., Int. Ed. 2010, 49, 868-872.

(32) Huang, J.; Kim, F.; Tao, A. R.; Connor, S.; Yang, P. Nat. Mater. 2005, 4, 896-900.

(33) Yao, J.; Yan, H.; Lieber, C. M. A. Nat. Nanotechnol. 2013, 8, 329-335.

(34) Ozin, G. A.; Hou, K.; Lotsch, B. V.; Cademartiri, L.; Puzzo, D. P.; Scotognella, F.; Ghadimi, A.; Thomson, J. Mater. Today 2009, 12, $12-23$.

(35) Diao, Y.; Tee, B. C. K.; Giri, G.; Xu, J.; Kim, D. H.; Becerril, H. A.; Stoltenberg, R. M.; Lee, T. H.; Xue, G.; Mannsfeld, S. C. B.; et al. Nat. Mater. 2013, 12, 665-671.

(36) Han, W.; Lin, Z. Q. Angew. Chem., Int. Ed. 2012, 51, 15341546.

(37) Yabu, H.; Shimomura, M. Adv. Funct. Mater. 2005, 15, 575-581.

(38) Hong, S. W.; Du, F.; Lan, W.; Kim, S.; Kim, H.-S.; Rogers, J. A. Adv. Mater. 2011, 23, 3821-3826.

(39) Leizerson, I.; Lipson, S. G.; Lyushnin, A. V. Langmuir 2004, 20, 291-294.

(40) Allemand, J. F.; Bensimon, D.; Jullien, L.; Bensimon, A.; Croquette, V. Biophys. J. 1997, 73, 2064-2070.

(41) Zhang, J.; Ma, Y.; Stachura, S.; He, H. Langmuir 2005, 21, $4180-4184$

(42) Kim, J. H.; Shi, W.-X.; Larson, R. G. Langmuir 2007, 23, 755764.

(43) Nakao, H.; Taguchi, T.; Shiigi, H.; Miki, K. Chem. Commun. 2009, 1858-1860.

(44) Jin, Z.; Sun, W.; Ke, Y.; Shih, C.-J.; Paulus, G. L. C.; Hua Wang, Q.; Mu, B.; Yin, P.; Strano, M. S. Nat. Commun. 2013, 4, 1663.

(45) Xia, F.; Farmer, D. B.; Lin, Y.-m.; Avouris, P. Nano Lett. 2010, $10,715-718$

(46) Xu, W.; Seo, H.-K.; Min, S.-Y.; Cho, H.; Lim, T.-S.; Oh, C.-Y.; Lee, Y.; Lee, T.-W. Adv. Mater. 2014, 26, 3459-3464.

(47) Cheng, Z.; Zhou, Q.; Wang, C.; Li, Q.; Wang, C.; Fang, Y. Nano Lett. 2011, 11, 767-771.

(48) Lin, Y. C.; Lu, C. C.; Yeh, C. H.; Jin, C. H.; Suenaga, K.; Chiu, P.-W. Nano Lett. 2012, 12, 414-419.

(49) Suk, J. W.; Lee, W. H.; Lee, J.; Chou, H.; Piner, R. D.; Hao, Y.; Akinwande, D.; Ruoff, R. S. Nano Lett. 2013, 13, 1462-1467.

(50) Kim, R.-H.; Bae, M.-H.; Kim, D. G.; Cheng, H.; Kim, B. H.; Kim, D.-H.; Li, M.; Wu, J.; Du, F.; Kim, H.-S.; Kim, S.; Estrada, D.; Hong, S. W.; Huang, Y.; Pop, E.; Rogers, J. A. Nano Lett. 2011, 11, $3881-3886$

(51) Schwierz, F. Nat. Nanotechnol. 2010, 5, 487-496.

(52) Hwang, W.-S.; Tahy, K.; Zhao, P.; Nyakiti, L. O.; Wheeler, V. D.; Myers-Ward, R. L.; Eddy, C. R., Jr.; Gaskill, D. K.; Xing, H.; 
Seabaugh, A.; Jena, D. J. Vac. Sci. Technol. B 2014, 32, 012202/1012202/5.

(53) Deshpande, A.; Bao, W.; Miao, F.; Lau, C. N.; LeRoy, B. J. Phys. Rev. B: Condens. Matter Mater. Phys. 2009, 79 (205411), 1-6.

(54) Dorgan, V. E.; Bae, M.-H.; Pop, E. Appl. Phys. Lett. 2010, 97, 082112.

(55) Xie, G.; Shi, Z.; Yang, R.; Liu, D.; Yang, W.; Cheng, M.; Wang, D.; Shi, D.; Zhang, G. Nano Lett. 2012, 12, 4642-4646. 